\title{
Remitting Seronegative symmetrical synovitis with pitting edema syndrome in association with saxagliptine; case report
}

\author{
Muge Bilge $\mathrm{MD}^{1}$, Mine Adas MD², Recep Yilmaz Bayraktarli³ ${ }^{3}$ Aysen Helvaci MD ${ }^{1}$ \\ ${ }^{1}$ Okmeydani Education and Research Hospital, Internal Medicine, Istanbul, Turkiye \\ ${ }^{2}$ Okmeydani Education and Research Hospital, Endocrinology, Istanbul, Turkiye \\ ${ }^{3}$ Okmeydani Education and Research Hospital, Radiology, Istanbul, Turkiye
}

BACKGROUND: Remitting seronegative symmetrical synovitis with pitting oedema (RS3PE syndrome) is a very rare clinical entity including a tenosynovitis of the hands and wrists, and marked pitting edema on upper and lower extremities. RS3PE has been suggested to be associated with many disease conditions and drugs. Saxagliptine is DPP4 inhibitor that relatively new oral hypoglycemic agent. This case is the second report of the occurrence of RS3PE in patients receiving a DPP4 inhibitor.
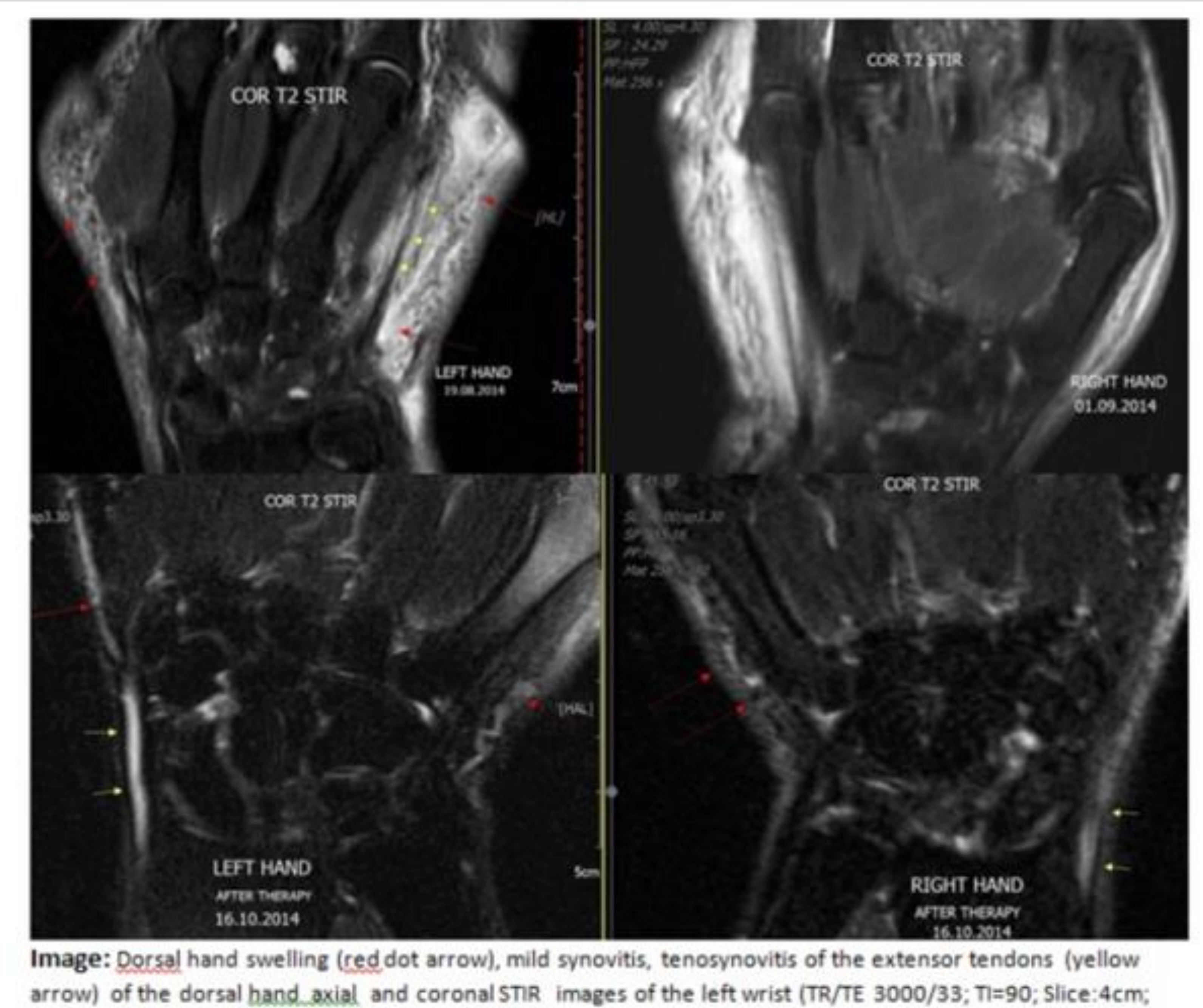
matrix $=256 \times 192)$. After therapx (second line picss).
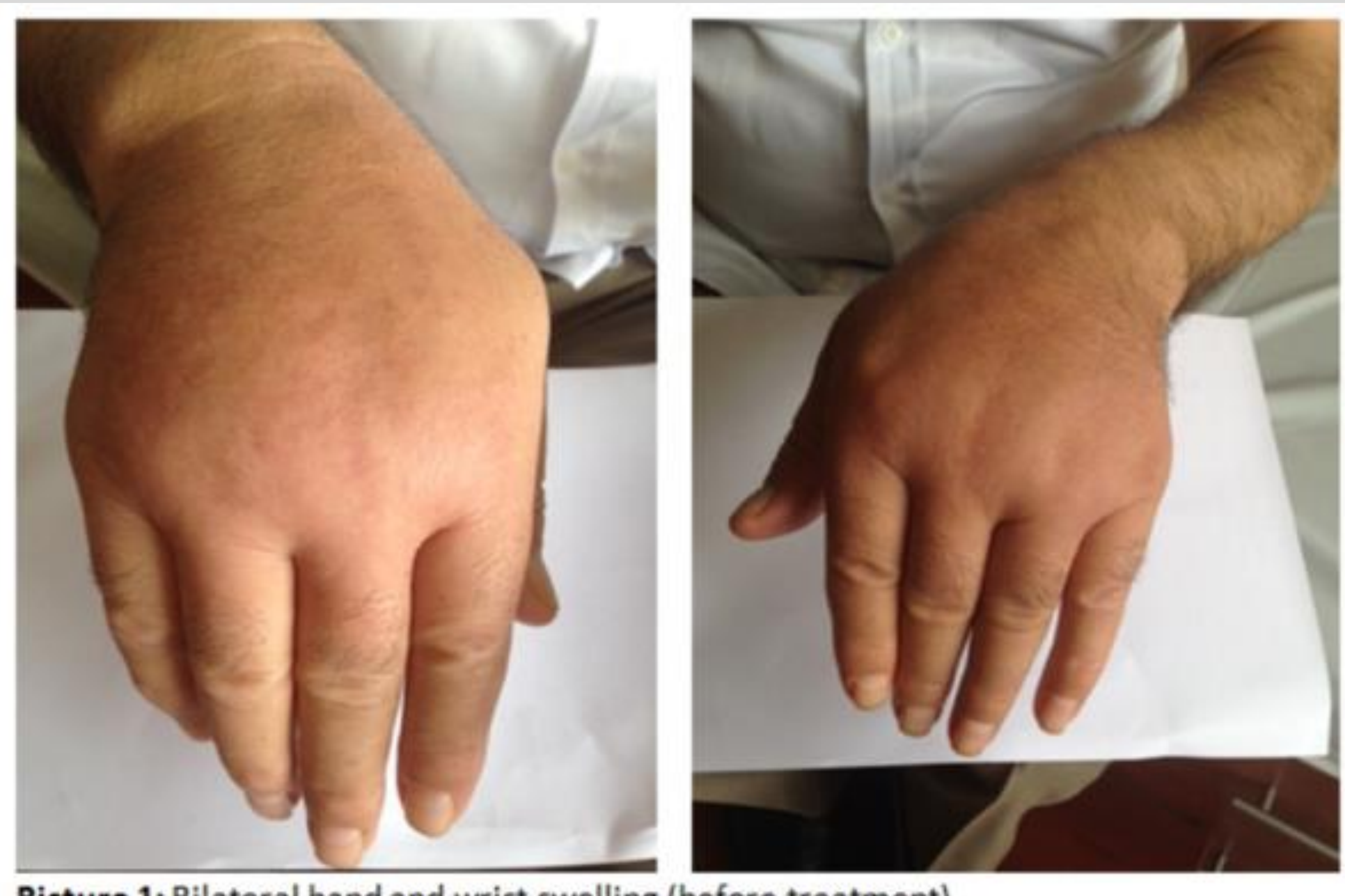

Picture 1: Bilateral hand and wrist swelling (before treatment)

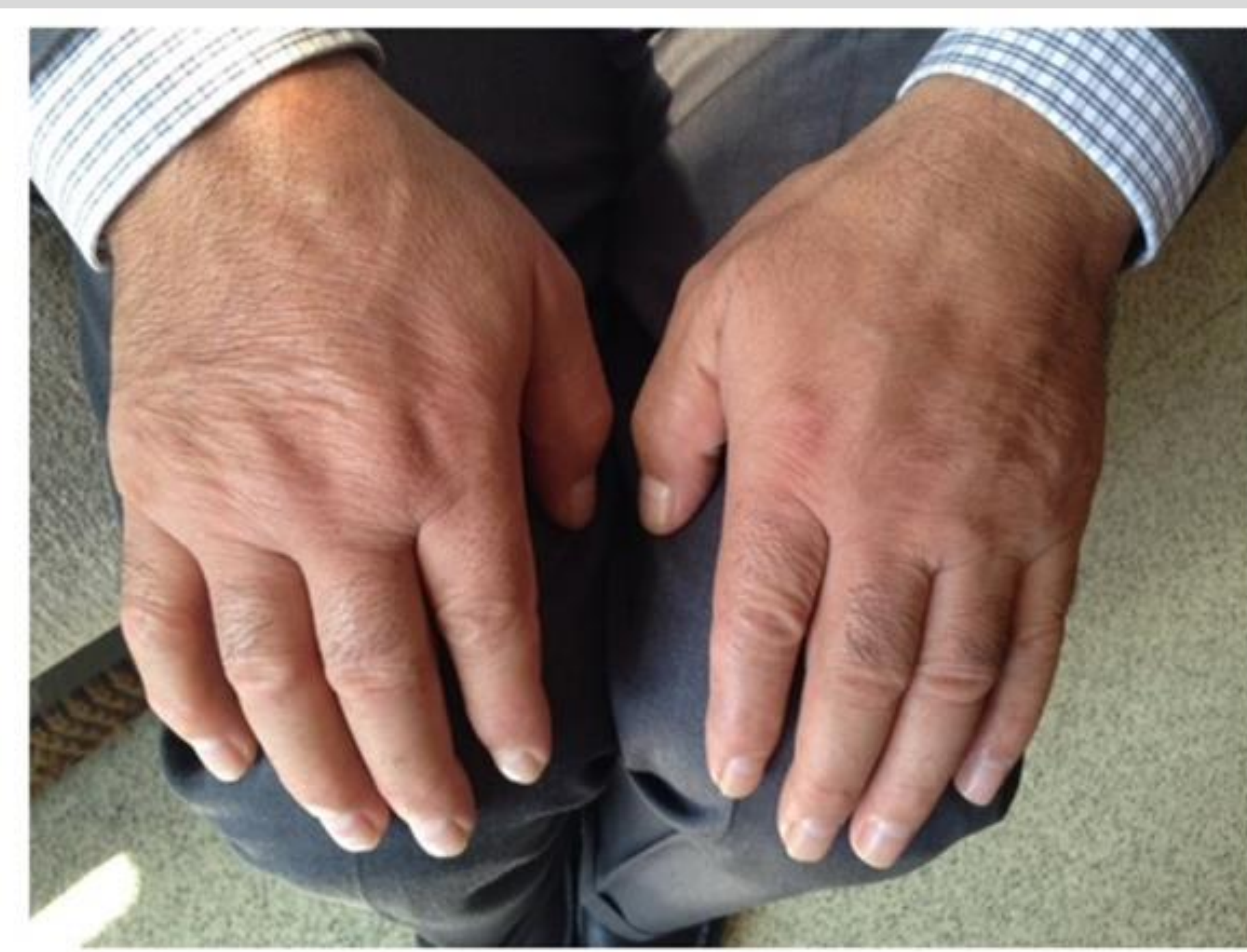

Picture 2: After treatment
CASE: A 51-year-old man presented with bilateral hand edema. The symptoms onset within after initiating saxagliptine of the treatment. He had type 2 diabetes mellitus (HA1C=6,6\%), and hyperlipidemia. At the time of presentation, his other medications were metformin, premixed insülin (aspart, protamine), and rosuvastatine. He did not have any history of drug allergy or systemic rheumatic illness. Physical examination revealed tenosynovitis characterized by swelling and moderate tenderness of the metacarpophalangeal and proximal interphalangeal joints bilaterally. There was marked non-tender pitting edema on the dorsum of both hands and wrist joints. Physical exam results were otherwise normal. Laboratory evaluation revealed normal ESR, and negative ANA, ANCA, RF, ACPA. Xrays and $M R I$ revealed only soft-tissue swelling without evidence of joint destruction. Due to the absence of complete remission with removal of saxagliptine during the four weeks, saxagliptine was tought to be responsable for the etiology of RS3PE syndrome. Than patient was treated with prednisone $20 \mathrm{mg}$ daily. Clinical findings were dramatic response to prednisone.

CONCLUSIONS: This case demonstrates a possible direct aetiological link between saxagliptine and RS3PE. 